\title{
Spatial-frequency-contingent color aftereffects: Adaptation with one-dimensional stimuli
}

\author{
R. H. DAY, WILLIAM R. WEBSTER, and OWEN GILLIES \\ Monash University, Clayton, Victoria, Australia \\ and \\ BORIS CRASSINI \\ Deakin University, Victoria, Australia
}

\begin{abstract}
The McCollough effect was shown to be spatial-frequency selective by Lovegrove and Over (1972) after adaptation with vertical colored square-wave gratings separated by 1 octave. Adaptation with slide-presented red and green vertical square-wave gratings separated by 1 octave failed to produce contingent color aftereffects (CAEs). However, when each of these gratings was adapted alone, strong CAEs were produced. Adaptation with vertical colored sine-wave gratings separated by 1 octave also failed to produce CAEs, but strong effects were produced by adaptation with each grating alone. By varying the spatial frequency of the test sine wave, CAEs were found to be tuned for spatial frequency at 2.85 octaves after adaptation of 4 cycles per degree (cpd) and at 2.30 octaves after adaptation of $8 \mathrm{cpd}$. Adaptation of both vertical and horizontal sine-wave gratings produced strong CAEs, with bandwidths ranging from 1.96 to 2.90 octaves and with lower adapting contrast producing weaker CAEs. These results indicate that the McCollough effect is more broadly tuned for spatial frequency than are simple adaptation effects.
\end{abstract}

If, for several minutes, an observer repeatedly views a sequence consisting of a vertical grating in red light and a horizontal one in green light, subsequent achromatic versions of the gratings will be tinged with pink on the horizontals and with green on the verticals (McCollough, 1965; Stromeyer, 1978). Colored edge detectors were first suggested as the basis for this adaptation effect (McCollough, 1965). Lovegrove and Over (1972), in a much cited paper, proposed an alternative mechanism after they found that a color aftereffect (CAE) could also be made contingent on both spatial frequency and color. They found selective-color CAEs on vertical square-wave gratings differing in color and separated by 1 octave in spatial frequency, and Wyatt (1974) later reported similar results. Lovegrove and Over (1972) interpreted these outcomes in terms of spatial-frequency channels similar to those proposed by Blakemore and Campbell (1969). The latter found that adaptation of achromatic sine-wave gratings had an adaptation tuning bandwidth of approximately 1 octave at half amplitude. The exact specification of the bandwidth of spatial-frequency filters is an issue of some controversy (Kelly \& Burbeck, 1985). Other adaptation studies (Blakemore, Muncey, \& Ridley 1973; Swift \& Smith, 1982) and a subthreshold additivity study (Sachs,

The authors wish to thank Keith Willenberg and John Cassell for computer programming. We also wish to thank V. Kohout for photography, Rosemary Williams for illustrations, and, in particular, Moyra Farrington for typing. This research was supported by a grant from the Australian Research Committee. Correspondence should be addressed to William R. Webster, Department of Psychology, Monash University, Clayton, Victoria, 3168, Australia.
Nachmias, \& Robson, 1971) have shown very narrow bandwidths, whereas others using a masking procedure have shown broad bandwidths (Henning, Hertz, \& Broadbent, 1975). It has been suggested by Kelly and Burbeck (1985) that approximately $0.75-1.0$ octave would appear to be the best fit to bandwidths associated with some visual receptive fields, although R. L. De Valois, Albrecht, and Thorell (1982) reported a median bandwidth of $1.40 \mathrm{oc}-$ taves for cells in primate visual cortex (VI). However, Lovegrove and Over (1972) did not point out that Blakemore and Campbell (1969) had also reported that adaptation of achromatic square waves produced much broader tuning than 1 octave, because adaptation influenced the third harmonic. Furthermore, Stromeyer (1972) found that the McCollough effect with colored square waves was tuned as broadly as 3-4 octaves. Breitmeyer and Cooper (1972) suggested that a separation of 2 octaves was required to produce selective-color CAEs with square-wave gratings. Thus, it is difficult to understand how strong selective adaptation was obtained with two square-wave gratings separated by only 1 octave. The central aim of the experiments reported here was to investigate and establish the tuning of the McCollough effect for spatial frequency with one-dimensional grating stimuli.

\section{EXPERIMENT 1}

Lovegrove and Over (1972) and Wyatt (1974), presented gratings by slide projection. The contrast of the patterns approached $100 \%$ in Wyatt's (1974) study but was not specified by Lovegrove and Over (1972). Our first experiment was concerned with checking the results 
of those experiments by also presenting square-wave gratings by slide projection. From the work of Stromeyer (1972), we predicted that there would be little selective adaptation to square-wave gratings that differed in color and also in spatial frequency by 1 octave. That is, there would be cancellation of the aftereffect since both gratings would stimulate different color detectors, which were in overlapping bandpasses for spatial frequency. Thus, when achromatic test patterns were presented, they would simultaneously activate both groups of detectors. To test this, subjects were adapted with gratings separated by 1 octave and with the two gratings presented alone. The prediction was that little, if any, CAE would occur when both gratings were adapted, but a strong CAE would be generated by adaptation of each alone.

\section{Methods}

Subjects. Thirty undergraduates with normal color vision, according to the Ishihara (1964) test, participated. All were naive in regard to the purpose of the experiment.

Apparatus and Stimulus patterns. All adaptation and testing procedures were carried out in a darkened laboratory. The stimulus patterns were nine photographic slides, which were projected onto a white screen by a projector (Pradovit, color 250). All stimulus patterns measured $13^{\circ} \times 13^{\circ}$ on the screen. There were three experimental conditions, consisting of two adapting stimuli and one test stimulus. The adapting patterns were either a red (Wratten filter 26) and black vertical square-wave grating ( $4.5 \mathrm{~cd} / \mathrm{m}^{2}$, contrast $\left.40 \%\right)$ or a green (Wratten filter 41 ) and black vertical square-wave grating $\left(7.2 \mathrm{~cd} / \mathrm{m}^{2}\right.$, contrast $\left.40 \%\right)$ separated by 1 octave in spatial frequency. The test pattern consisted of a split-field pattern of achromatic vertical square waves $\left(18.0 \mathrm{~cd} / \mathrm{m}^{2}\right.$, contrast $\left.70 \%\right)$. The left field consisted of achromatic square-wave gratings of the lower frequency of each pair of inducing gratings and the right field of squarewave gratings of the higher frequency of the pair. The adapting gratings were of low spatial frequency ( 1 and 2 cycles per degree [cpd]) in Condition 1, of medium frequency ( 4 and $8 \mathrm{cpd}$ ) in Condition 2 , and of high frequency ( 8 and $16 \mathrm{cpd}$ ) in Condition 3 . The adapting patterns were counterbalanced for color in each experimental condition. Contrast was defined as $L_{\max }-L_{\min } / L_{\max }+L_{\min }$, where $L_{\max }=$ maximum luminance and $L_{\min }=$ minimum luminance as measured with a Minolta Autospot II photometer. The subjects were seated $6 \mathrm{~m}$ from the screen, a distance at which the required range of spatial frequencies occurred.

Procedure. Ten subjects were assigned to each of the three frequency conditions. All subjects were tested in three sessions, with 1-week intervals between sessions to allow any aftereffect to fully dissipate. In the first session, the subjects were adapted with both gratings. In the second, they were adapted with the higher spatialfrequency grating in alternation with a blank screen $\left(1.3 \mathrm{~cd} / \mathrm{m}^{2}\right)$. In the last session, the subjects were adapted with the lower spatialfrequency pattern, again in alternation with a blank screen. Each pattern was presented in alternation for $10 \mathrm{sec}$ over a 20 -min adaptation period. Before each adaptation session, there was a pretest in which subjects made a forced-choice decision as to whether each half-field of the achromatic stimulus was red, green, or white. The order in which each half field was judged was counterbalanced across subjects. The subjects were instructed to keep the head upright and steady and not to fixate the patterns but to move their gaze freely over them. After adaptation, $2 \mathrm{~min}$ were allowed to elapse so that any afterimage could dissipate. Then, each subject was again asked to make a forced choice about the color of each half of the split- field pattern. When color was reported, the subject was asked to rotate the head laterally through $90^{\circ}$ to establish whether the color disappeared. This procedure provided a test for an orientationcontingent effect. All subjects were asked if they could discriminate between each pair of gratings on the basis of both color and frequency.

\section{Results and Discussion}

The data were analyzed by the McNemar test for significance of change between the pretest and posttest results, which provided a measure of the CAE. This change did not reach significance for any of the frequency conditions after adaptation with two gratings. When two gratings were adapted, most subjects reported white on both halves of the stimulus pattern. The few remaining subjects reported positive effects on both halves. For this reason, we combined the scores for both gratings for each frequency pair. In every case in which one grating was adapted, a significant proportion of the subjects exhibited an aftereffect (Figure 1). In fact, the minimum proportion was 9 out of 10 subjects exhibiting the CAEs. Thus, selective CAEs with gratings separated by 1 octave did not occur under our conditions. However, the experimental conditions were appropriate for the occurrence of CAEs, since they were strongly induced for each separate grating at all spatial frequencies. Rotating the head through $90^{\circ}$ resulted in the abolition of the effect, thus indicating that it was not due to long-lasting weak afterimages. All subjects reported that they could discriminate between the two test gratings after adaptation.

The results of Experiment 1 show that adaptation with two vertical square-wave gratings under these conditions does not give rise to selective-color CAEs when the adapting gratings are separated by only 1 octave in spatial frequency. Yet, strong McCollough effects were produced when each adapting grating was presented alone. Aftereffects were found at all spatial frequencies. These results support the prediction that when two square-wave gratings, an octave apart, are adapted in alternation, interference $\alpha c$ curs between the overlapping mechanisms underlying the adaptation of each. The results also confirm Breitmeyer and Cooper's (1972) suggestion that square-wave gratings separated by at least 2 octaves are necessary to produce selective spatial-frequency CAEs. However, an edgedetection model might also explain the results. If the different color gratings stimulated edge detectors of the same size, then it could be argued that similar cancellation effects would be produced by achromatic gratings. Thus, the results of Experiment 1 do not differentiate between the edge-detector theory and spatial-filter theory of spatial vision, but they do indicate that if the $\mathrm{McCol}-$ lough effect is contingent for spatial frequency, then its spatial frequency is more broadly tuned than 1 octave for square-wave gratings.

A more rigorous test of the two theories would be provided if sine-wave gratings were employed to derive a measure of bandpass tuning. This procedure would provide more appropriate stimulus conditions for a compar- 


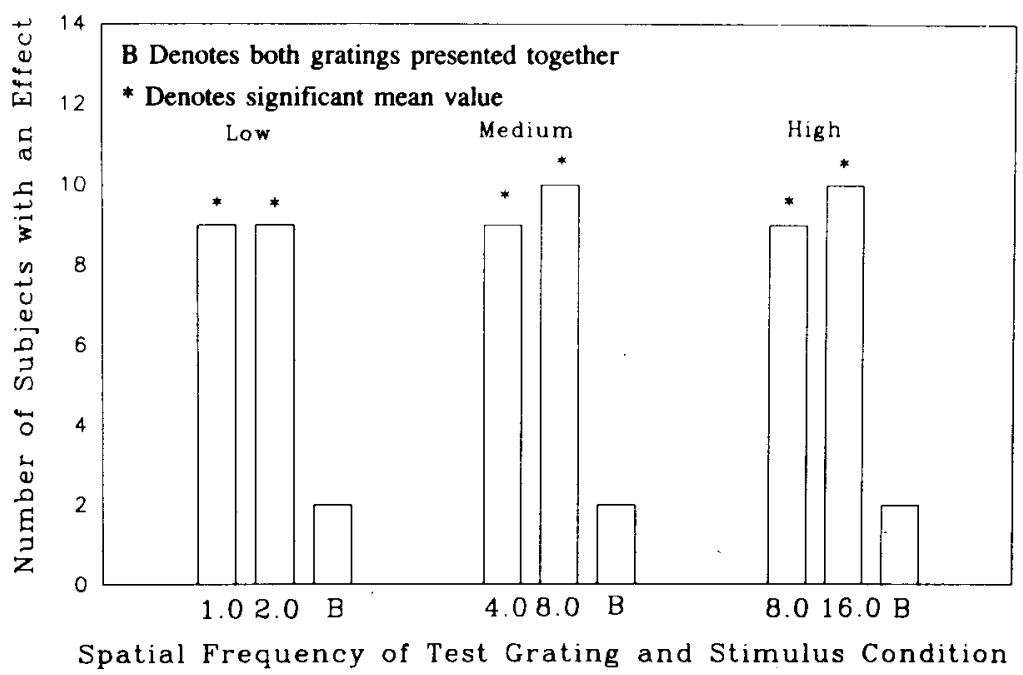

Figure 1. Histograms showing the number of subjects reporting a McCollough effect when the gratings were either adapted together (B) or separately. The results are presented for three adapting-frequency conditions: low (1.0 and $2.0 \mathrm{cpd})$, medium (4.0 and $8.0 \mathrm{cpd})$, and high $(8.0$ and $16.0 \mathrm{cpd})$. Ten subjects were adapted and tested under each condition.

ison of CAE tuning with the tuning of the simple adaptation situation (Blakemore \& Campbell, 1969).

\section{EXPERIMENT 2}

Simple adaptation studies with threshold judgments (Blakemore \& Campbell, 1969) and suprathreshold judgments (Blakemore et al., 1973) have shown that the spatial-frequency tuning to sine-wave gratings is in the range of 0.75 to 1.0 octave. Therefore, the aim of Experiment 2 was to induce the McCollough effect with colored sine-wave gratings and to examine tuning with achromatic sine-wave gratings. Blakemore and Campbell's (1969) experiments suggested that sine-wave gratings higher than $3 \mathrm{cpd}$ are necessary to produce narrow bandpass tuning centered around the adapting frequency. They suggested that below $3 \mathrm{cpd}$, another mechanism might operate, since low-frequency patterns exhibited maximum adaptation around $3 \mathrm{cpd}$ instead of around the low frequency used for adaptation. While Jones and Tulunay-Keesey (1975) have reported that this lowfrequency effect might be an artifact of the number of bars on the screen, we decided to test the spatial-frequency hypothesis with sine-wave gratings of 4 and $8 \mathrm{cpd}$ to prevent any possible confounding. The same experimental design as that of Experiment 1 was used. Each subject was adapted with both gratings and then with each grating alone. A computer graphics system lent itself to the presentation of a range of test gratings for the measurement of spatial-frequency tuning.

\section{Methods}

Apparatus and Stimulus patterns. Eight undergraduate students were tested. All were screened for color defects using the Ishihara (1964) test as before. Three of the subjects required corrective lenses and wore them during the experiment. All subjects were naive as to the aim of the experiment. The stimulus patterns were presented on a Barco color monitor (Model CD33), using a real-time imageprocessing system (Matrox video digitizer, Model PIP-EZ) interfaced to an IBM clone (Super PC, Model AT 286). Each of the patterns subtended a visual angle of $8^{\circ}$ vertically and $7.5^{\circ}$ horizontally. The two adapting patterns were vertical sine-wave gratings of 4 and $8 \mathrm{cpd}$, respectively. One was red and black (dominant wavelength $620 \mathrm{~nm}, 10 \mathrm{~cd} / \mathrm{m}^{2}$, contrast $80 \%$ ) as measured by a Minolta Chromameter (Model CL 100); the other was green and black (dominant wavelength $515 \mathrm{~nm}, 18 \mathrm{~cd} / \mathrm{m}^{2}, 80 \%$ contrast). There were seven achromatic sine-wave test patterns $\left(23.4 \mathrm{~cd} / \mathrm{m}^{2}, 60 \%\right.$ contrast) with spatial frequencies of $1,2,4,6,8,16$, and $20 \mathrm{cpd}$. The subjects sat $2 \mathrm{~m}$ from the screen in the darkened laboratory, as before, and were instructed to keep their heads upright and steady and not to fixate but to move their gaze over the adapting patterns.

Calibrations. The subjects could alter or cancel the color appearance of the achromatic test gratings by pressing one of the three colored buttons (red, green, or white) that controlled through the computer the combined output of the red (R), green (G), and blue (B) guns of the monitor. It was shown earlier for a similar computercontrolled display that each buttonpress produced a linear step in color space (Webster, Crassini, \& Willenberg, 1987; Webster, Day, \& Willenberg, 1988). That is, if red was added to an achromatic stimulus by a buttonpress, then the position in color space moved by one linear step toward red. The position in color space covered by the cancellation process ranges from $x=0.259, y=0.329$ (green) to $x=0.290, y=0.257$ (red), with white as $x=0.269$, $y=0.290$ (see Webster et al., 1988, for details of color space and linearity of cancellation). A change could not be detected with one buttonpress. In a preliminary study, 27 subjects were given eight split-field stimulus patterns in random order. One half field was set at $0,5,10$, or 15 computer steps from white. Thus, one homogeneous half field would be red, green, or white, according to the computer step set. The other half field was homogeneous white. All subjects were asked to press the appropriate button to cancel the color, so that both half fields matched. The order of setting was counterbalanced across the halves of the field. All subjects were asked to press the appropriate button to cancel the color. The subjects were quite accurate in canceling any color to white 
Table 1

Number of Buttonpresses to Cancel to White

\begin{tabular}{crrr}
$\begin{array}{c}\text { Number of } \\
\begin{array}{c}\text { Computer Steps } \\
\text { from White }\end{array}\end{array}$ & \multicolumn{1}{c}{$\boldsymbol{M}$} & $S D$ & \multicolumn{1}{c}{$t$ Value } \\
\hline 0 & -0.14 & 2.35 & $t(26)=-0.45, p>.05$ \\
5 & 5.17 & 2.45 & $t(26)=+0.54, p>.05$ \\
10 & 9.54 & 2.45 & $t(26)=-1.41, p>.05$ \\
15 & 14.49 & 2.49 & $t(26)=-1.55, p>.05$ \\
\hline
\end{tabular}

(Table 1); that is, if the subjects were given a color that was 10 computer steps from white, then they would take on the average 10 buttonpresses (i.e., the same number of computer steps) to cancel the color to white. The standard deviations for each condition are remarkably similar, indicating the same variation in responding no matter what starting position was set. Dependent $t$ tests revealed no difference between the number of buttonpresses and the number of computer steps to reach white at each setting. It was concluded, therefore, that the system provided a reliable measure of color cancellation and that each subject was nulling to white in a similar way.

Procedure. Each subject was tested as before in three sessions, with 1-week intervals between sessions. Each session consisted of a pretest with the seven achromatic gratings, followed by adaptation and then a posttest with the same the seven gratings. The test gratings were presented in random order. Between the adaptation and the posttests, a period of $2 \mathrm{~min}$ was again allowed to elapse so that any afterimages could dissipate. In the first session (Condition 1), the 4- and 8-cpd gratings were presented in alternation for 10 -sec periods. Thirty pairs were presented, resulting in an adaptation period of $10 \mathrm{~min}$. The color of the adapting stimuli was counterbalanced across subjects. In the second session (Condition 2), the 4-cpd grating was presented for $10 \mathrm{sec}$, alternating with a 10 -sec blank screen $\left(0.77 \mathrm{~cd} / \mathrm{m}^{2}\right)$ for $10 \mathrm{~min}$. The color of the gratings was counterbalanced, with the restriction that if a subject viewed a red 4-cpd grating in Condition 1, the same grating would be adapted in Condition 2 . In the third session (Condition 3), the 8-cpd grating was presented for $10 \mathrm{sec}$, alternating with a 10 -sec blank screen for 10 minutes. Counterbalancing for color was the same as that in the second session.

Scoring. For each condition, the score was the number of buttonpresses to cancel to white. In Conditions 2 and 3, the cancellation of a color complementary to that of the adapting grating was scored as positive and cancellation of one that was the same as the adapting grating was scored as negative. In Condition 1, cancellation of a color complementary to that of the 4-cpd grating was scored as positive for gratings of $4 \mathrm{cpd}$ and less. For gratings of $8 \mathrm{cpd}$ and greater, the opposite color was scored as positive. For 6-cpd gratings, the color complementary to the 4-cpd grating was arbitrarily scored as positive.

\section{Results and Discussion}

The data for each condition were analyzed with a repeated measures two-way analysis of variance (ANOVA) on the difference scores, defined as before. Subsequently, dependent $t$ tests were carried out on these scores. The ANOVA for Condition 1 failed to reveal a significant McCollough effect $[F(7,49)=1.57, p>.05]$ after adaptation with two gratings (Figure 2). The analysis of the individual means showed that no posttest was significantly different from its pretest mean (Table 2). The ANOVA for Condition 2 showed that a significant McCollough effect occurred across grating frequency $[F(7,49)=3.04$, $p<.01]$ after adaptation to a 4-cpd grating. The mean scores are plotted in Figure 2. The means for gratings of $2,4,6,8$, and $16 \mathrm{cpd}$ differed significantly from their pretest means (Table 2). Similar results were obtained for Condition 3, with a significant McCollough effect across gratings $[F(7,49)=6.72, p<.01]$ after adaptation to an 8-cpd grating. The mean scores are plotted as a function of spatial frequency in Figure 2. The means for gratings of 4, 6, 8, and 16 cpd differed significantly from the pretest scores (Table 2). Adaptation with a 4-cpd grating had a bandwidth of 2.85 octaves at half amplitude; adap-

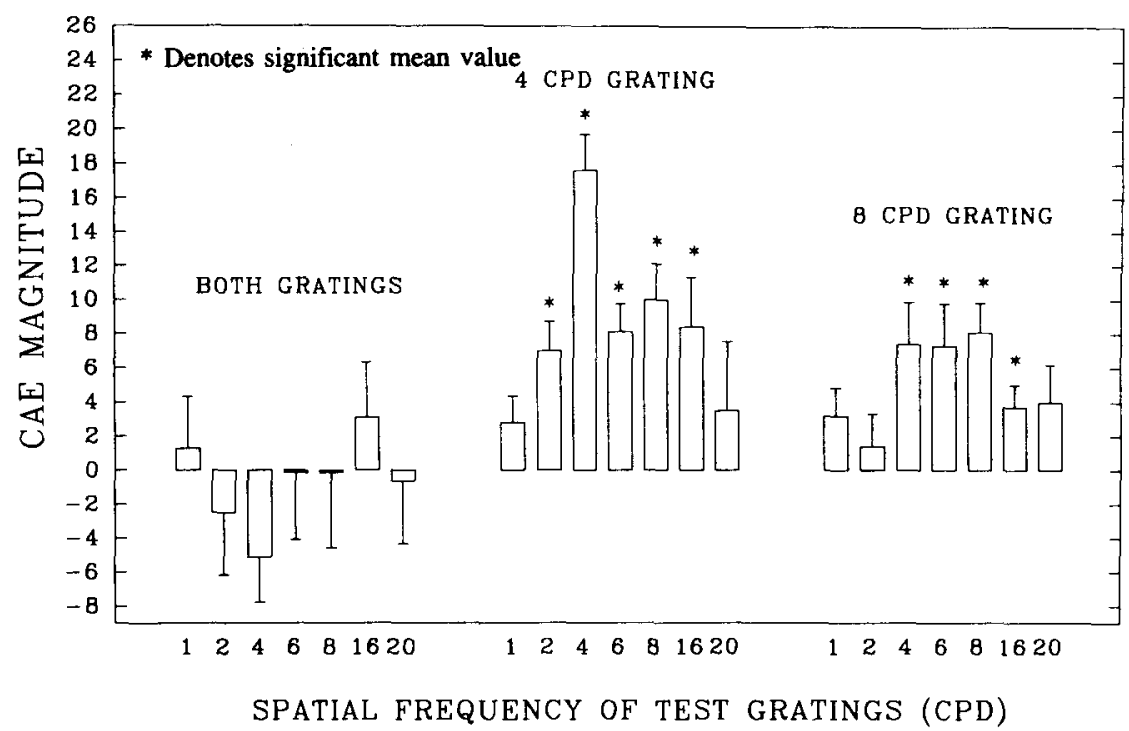

Figure 2. Plots of mean CAE magnitudes as a function of spatial frequency of test gratings. The scores are plotted for three adapting conditions: both gratings ( 4 and 8 cpd), 4-cpd grating alone, and 8-cpd grating alone. Error bars represent the standard error of the mean. 
Table 2

Mean CAE Scores, Standard Deviations, and $t$ Values for Experiment 2

\begin{tabular}{|c|c|c|c|c|c|c|c|c|c|}
\hline \multirow{3}{*}{$\begin{array}{c}\text { Spatial Frequency (cpd) } \\
\text { of Test Stimuli }\end{array}$} & \multicolumn{9}{|c|}{ Adapting Stimuli } \\
\hline & \multicolumn{3}{|c|}{ 4- and 8-cpd Gratings } & \multicolumn{3}{|c|}{ 4-cpd Grating } & \multicolumn{3}{|c|}{ 8-cpd Grating } \\
\hline & $M$ & $S D$ & $t$ & $M$ & $S D$ & $t$ & $M$ & $S D$ & $t$ \\
\hline 1 & 1.25 & 8.58 & 0.41 & 2.75 & 4.37 & 1.78 & 3.25 & 4.65 & 1.98 \\
\hline 2 & -2.50 & 10.41 & -0.68 & 7.00 & 4.95 & $3.99 *$ & 1.38 & 5.50 & 0.71 \\
\hline 4 & -5.13 & 7.61 & -1.91 & 17.63 & 5.97 & $8.34 *$ & 7.38 & 7.13 & $2.93 \dagger$ \\
\hline 6 & -0.13 & 11.23 & -0.03 & 8.13 & 4.61 & $4.98^{*}$ & 7.25 & 7.15 & $2.87 \dagger$ \\
\hline 8 & -0.13 & 12.63 & -0.03 & 10.00 & 6.02 & $4.69^{*}$ & 8.13 & 4.99 & $4.60^{*}$ \\
\hline 16 & 3.13 & 9.14 & 0.97 & 8.38 & 8.37 & $2.83+$ & 3.75 & 3.85 & $2.76 t$ \\
\hline 20 & -0.63 & 10.51 & -0.17 & 3.50 & 11.41 & 0.87 & 4.00 & 6.35 & 1.78 \\
\hline
\end{tabular}

${ }^{*} p<.01 . \quad+p<.05$.

tation with an 8-cpd grating had a bandwidth of $2.30 \mathrm{oc}-$ taves. In each case, there was a significant CAE for gratings that were 1 octave away from the adapting grating (Figure 2). Thus, it would be expected that after adaptation to two different-colored gratings differing by $1 \propto c-$ tave, each achromatic test grating would elicit two opposing CAEs.

The results of Experiment 2 confirm and extend the results of Experiment 1. Even when sine-wave gratings were used as adapting patterns, it was not possible to generate selective McCollough effects with two gratings 1 octave apart in spatial frequency. However, when each grating was adapted alone, a strong CAE occurred. The bandwidth of the spatial-frequency tuning curve for each grating was smaller than those reported by Stromeyer (1972) for square-wave gratings but greater than the bandwidths reported for simple adaptation effects, which are in the range of 0.75 to 1.0 octaves for threshold and suprathreshold test stimuli (Blakemore et al., 1973). Thus, the results fail to confirm the conclusion reached by Lovegrove and Over (1972) that the McCollough effect is contingent for spatial frequency, with the bandwidth of the channels of the same order as those used to support the theory of spatial-frequency filters (Blakemore \& Campbell, 1969). These results do not imply that the McCollough effect is not contingent for spatial frequency. Rather, they suggest that the contingency is based on broad tuning. The experiments do not, however, address the issue of which pairs of frequencies would produce CAEs, but the tuning data would suggest that, even with sine-wave gratings, a separation of at least 2-3 octaves would be required.

An edge-detector theory could also explain these results, since simple adaptation with square waves (Blakemore \& Campbell, 1969) is more broadly tuned than 1.0 octave. The edge-detection model of Marr (1982) and Marr and Hildreth (1980) could also explain these data, because it is posited on a set of filters of different sizes before activation of an edge mechanism. It might also be possible to explain these tuning results by adaptation of nonperiodic detectors for bars or lines. That is, if there is a range of sizes of bar detectors, then these could account for the broad tuning data (Thomas, 1970). However, when bars are adapted, threshold elevation is uniform across both a range of bar widths and the spectrum of sine-wave gratings
(Sullivan, Georgeson, \& Oatley, 1972). That is, there is no selective adaptation and, hence, no tuning functions. Thus, it would seem that bandwidths found with simple adaptation and CAEs could not be produced by bar detectors.

\section{EXPERIMENT 3}

In Experiment 2, the spatial-frequency tuning for the McCollough effect was found to be broader than the tuning observed in simple adaptation experiments (Blakemore \& Campbell, 1969). However, the data was obtained using an experimental paradigm similar to that employed by Stromeyer (1972)-that is, only one grating was used. The purpose of Experiment 3 was to examine spatial-frequency tuning in the conventional CAE situation in which two sinewave gratings differing in both orientation and color but the same in spatial frequency were used for adaptation. It was possible that this might lead to narrower tuning. CAEs were generated with spatial frequencies of 2 and $4 \mathrm{cpd}$. The lower frequency was chosen because Blakemore and Campbell (1969) had reported that, for simple adaptation, they could not find bandpass tuning centered around the adapting frequency when frequencies below $3 \mathrm{cpd}$ were employed. All of these lower frequencies produced their maximum effect centered around $3 \mathrm{cpd}$. Blakemore and Campbell concluded that their spatial-filter theory applied only at frequencies above $3 \mathrm{cpd}$; they also concluded that, below $3 \mathrm{cpd}$, another mechanism might operate. (Blakemore \& Campbell had attempted to restrict stimulation to the fovea by means of a small stimulus area of $1.5^{\circ}$ in diameter.)

\section{Method}

Subjects. Two graduate students and 3 faculty members with normal acuity and color vision, according to the Ishihara (1964) test, served as subjects.

Apparatus and Stimulus patterns. Sine-wave gratings were presented on a Barco color monitor (Model GD 33), using the same program as in Experiment 2. The computer controlling the monitor was a DEC-LSI-11/73 interfaced with a real-time imageprocessing system (Image Technology Inc., Model AP-512). This system has been described before (Webster et al., 1987; Webster et al., 1988). The subjects used the same color-cancellation procedures as in Experiment 2. All subjects were tested in three experimental sessions, with 1 -week intervals between sessions. In the 
first session, each subject was adapted with a red and black vertical sine-wave grating of $2 \mathrm{cpd}\left(9.9 \mathrm{~cd} / \mathrm{m}^{2}, 80 \%\right.$ contrast) and a green horizontal sine-wave grating $\left(16.7 \mathrm{~cd} / \mathrm{m}^{2}, 80 \%\right.$ contrast $)$ of the same frequency. The pretest and posttest stimuli were seven achromatic sine-wave stimuli $\left(16.7 \mathrm{~cd} / \mathrm{m}^{2}, 30 \%\right.$ contrast). Each stimulus was a split-field pattern with a vertical sine wave in the left half field and a horizontal sine wave in the right half field. The spatial frequencies of the seven test patterns were $0.4,0.66,1.0,2.0,4.0$, 6.0 , and $10.0 \mathrm{cpd}$. In the second session, the adaptation stimuli were the same, but at $30 \%$ contrast. The pretest and posttest stimuli were the same as those in Session 1. In the third session, the adapting stimuli were a green and black vertical sine-wave grating of $4 \mathrm{cpd}$ $\left(9.9 \mathrm{~cd} / \mathrm{m}^{2}, 30 \%\right.$ contrast) and a red and black horizontal grating $\left(16.7 \mathrm{~cd} / \mathrm{m}^{2}, 30 \%\right.$ contrast) of the same frequency. There were eight split-field test stimuli similar to those of Sessions 1 and 2, but the spatial frequencies were $0.5,1.0,2.0,3.0,4.0,8.0,12.0$, and $16.0 \mathrm{cpd}$.

Procedure. Each session consisted of a pretest, an adaptation phase followed by a period $(2 \mathrm{~min}$ ) for the dissipation of any afterimages, and a posttest. Each adapting pattern was presented 30 times for $10 \mathrm{sec}$, resulting in an adaptation period of $10 \mathrm{~min}$. The general procedures for the presentation of the stimuli and the cancellation of color were the same as those in Experiment 2. The CAEs in the expected direction were scored as positive and those in the opposite direction as negative.

\section{Results and Discussion}

Again, for each subject, CAE scores were found by subtracting pretest from posttest estimates of CAEs. The data for vertical gratings from Sessions 1 and 2 were combined for analysis by a three-way within-subject ANOVA. A similar analysis was carried out on the horizontal gratings. For vertical gratings, the higher contrast gratings produced a larger CAE; the effect of contrast was not significant $[F(1,4)=5.17, \mathrm{p}>.05]$. The main effect of spatial frequency was significant $[F(6,24)=10.59, p<$ $.01]$. There was a significant interaction between contrast and spatial frequency $[F(6,74)=2.73, p<.05]$. The interaction can be seen in Figure 3. There was little difference between adaptation contrast for the lower spatial frequencies, but $80 \%$ contrast gave rise to stronger CAEs than did $30 \%$ contrast with higher spatial frequencies. For horizontal gratings, the main effect of contrast was significant $[F(1,4)=11.74, p<.05]$, as was the main effect of spatial frequency $[F(6,74)=8.51, p<.05]$. There was no significant interaction $[F(6,24)=1.09, p>.05]$.

Inspection of Figure 3 shows that contrast produced similar effects on both vertical and horizontal gratings. Not only did $80 \%$ contrast give rise to stronger CAEs for both vertical and horizontal test gratings than did $30 \%$ contrast, but the former produced broader tuning. The half-amplitude bandwidth for adapting at $80 \%$ contrast was 2.8 octaves for vertical gratings and 2.9 octaves for horizontal gratings. The $30 \%$ contrast adaptation produced bandwidths of 2.2 octaves for vertical gratings and 2.0 octaves for horizontal gratings. In three conditions, the maximum effect occurred at $4 \mathrm{cpd}$, instead of at the inducing frequency of $2 \mathrm{cpd}$.

The data for vertical adaptation from Session 3 were analyzed together with the horizontal data by a three-way repeated measures ANOVA. There was a significant main effect of spatial frequency $[F(7,28)=15.08, p<.05]$ but no difference between vertical and horizontal adaptation $[F(1,4)=4.40, p>.05]$ (see Figure 4 ). The halfamplitude bandwidth was 2.26 octaves for vertical gratings and 1.96 octaves for horizontal gratings.

The results of Experiment 3 confirm and extend the results of Experiment 2. The tuning of the McCollough effect after adaptation with two orthogonal sine-wave gratings was not narrower than the tuning found in Experiment 2 after adaptation with one grating. The tuning in
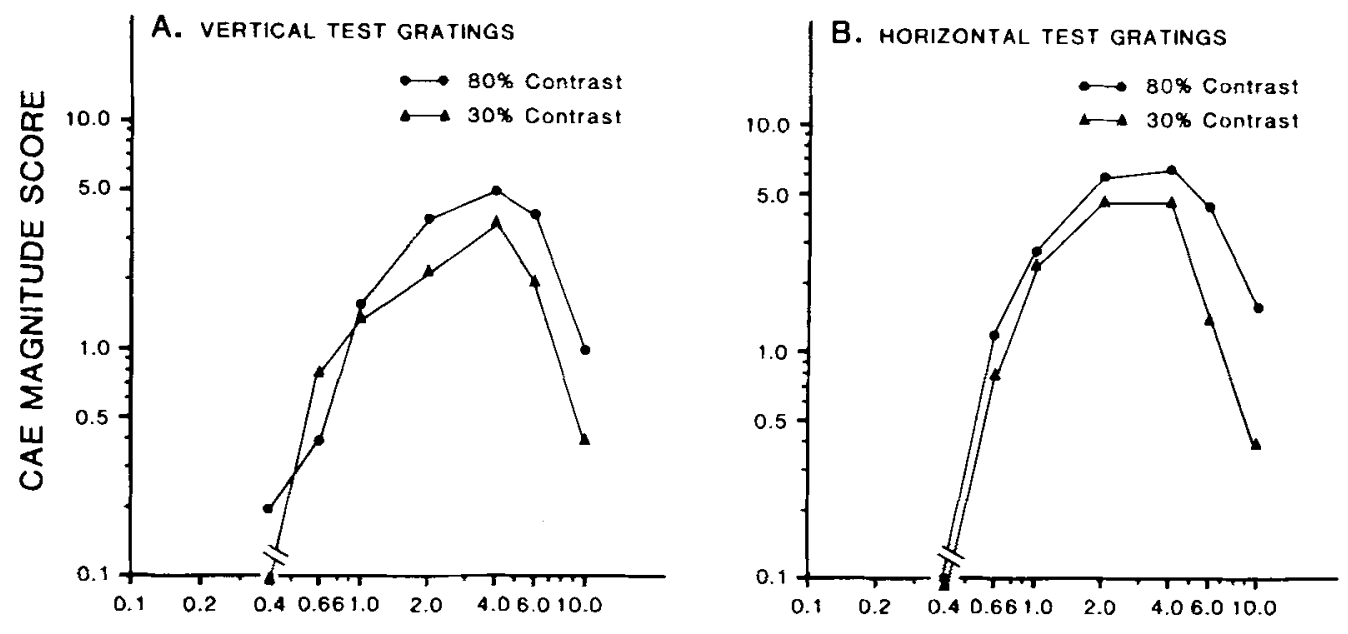

\section{SPATIAL FREQUENCY OF TEST GRATINGS \\ (cycles per degree)}

Figure 3. Mean CAE magnitudes plotted as a function of spatial frequency of sine-wave test stimuli at $80 \%$ and $30 \%$ contrast. All subjects were adapted with both vertical and horizontal colored sine-wave gratings of 2 cpd and either $80 \%$ or $30 \%$ contrast. Panel A shows the scores for vertical test gratings. Panel B shows the scores for horizontal test gratings. 


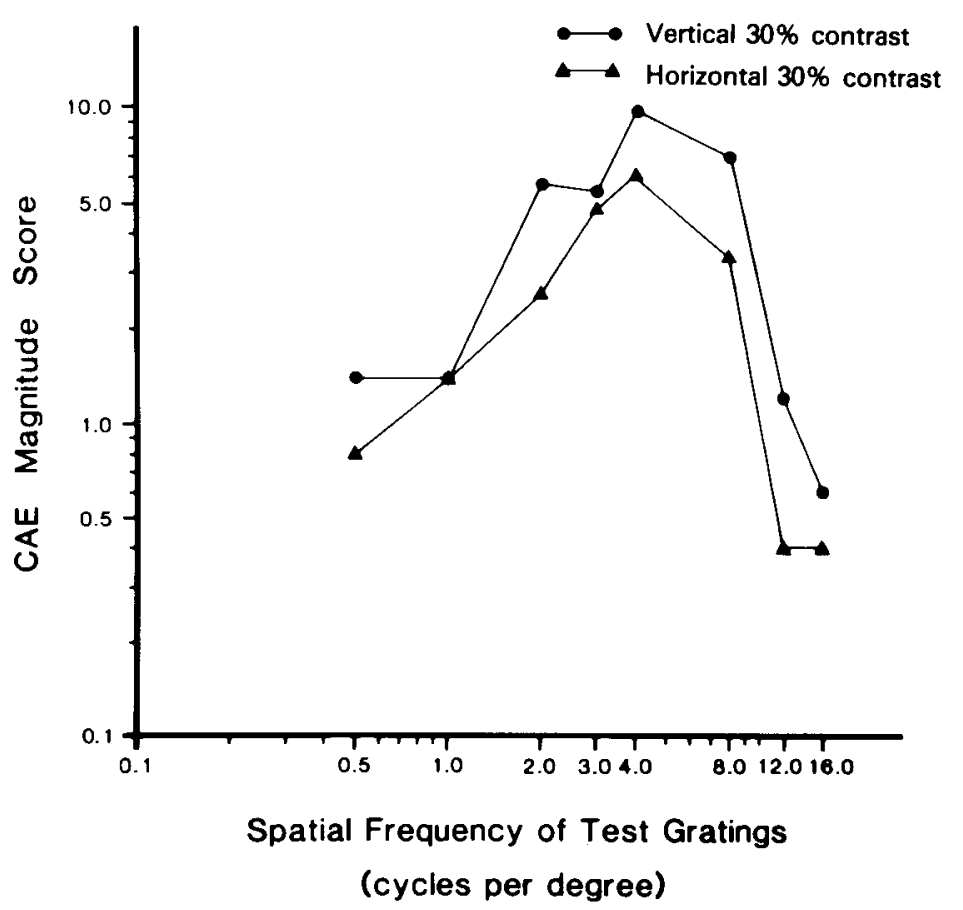

Figure 4. Mean CAE magnitudes plotted as a function of spatial frequency for vertical and horizontal sine-wave test gratings of $30 \%$ contrast. All subjects were adapted with colored vertical and horizontal sine-wave gratings of 4 cpd and $80 \%$ contrast.

each case was quite broad (2.0 to 2.9 octaves at half amplitude). The tuning was also sensitive to contrast, with narrower tuning at lower adapting contrast. Although the tuning was not as broad as that reported by Stromeyer (1972) after adaptation with square-wave gratings, it was broader than the tuning for threshold effects found following adaptation to achromatic sine-wave patterns (Blakemore \& Campbell, 1969; Swift \& Smith, 1982). This difference in tuning is not consistent with the hypothesis that the same spatially selective channels mediate both contingent and simple aftereffects. However, it is possible that the apparent difference in channel bandwidth is due to differences between both paradigms in terms of stimulus conditions and experimental procedures. One obvious difference is that the McCollough effect is commonly studied with suprathreshold stimulus patterns. However, narrow spatial tuning ( 0.75 octaves) has been reported for suprathreshold conditions for simple adaptation (Blakemore et al., 1973). This bandwidth was obtained after an equivalent contrast transformation was applied. The raw data had bandwidths of approximately 1.5 octaves, which is still smaller than those found for the McCollough effect. Our observations on the effect of contrast on the McCollough effect in these experiments agree with previous results (Stromeyer \& Dawson, 1978): stronger adapting contrast leads to a larger aftereffect. The shift in the locus of the maximum effect after adaptation with 2-cpd gratings could suggest that a mechanism similar to that proposed by Blakemore and Campbell (1969) operates with colored gratings of less than $3 \mathrm{cpd}$. There is no sign of this shift after adaptation to 4 and $8 \mathrm{cpd}$, although the effect at 1 octave away is quite marked in each case. Even lower frequencies need to be examined, but any lack in the number of cycles on the screen would not appear to be an explanation of the effect in our experiment.

\section{GENERAL DISCUSSION}

Our basic finding is that the McCollough effect is not contingent on spatial frequency to the degree proposed by Lovegrove and Over (1972). The broadness of tuning (2.0-2.9 octaves) suggests that the channels involved are other than those postulated from simple adaptation studies (Blakemore \& Campbell, 1969). In fact, the broadness of tuning suggests that the McCollough effect is not mediated by a spatial-frequency mechanism (R. L. De Valois \& K. K. De Valois, 1980; Marr \& Hildreth, 1980; Tyler, 1975). One proposal that can be made is that the broadness of tuning is a function of the sensitivity of channels to both color and spatial frequency. But simple adaptation studies of achromatic luminance gratings (Blakemore \& Campbell, 1969) and of colored luminance gratings (Bradley, Switkes, \& K. K. De Valois, 1988) reveal similar bandwidths. Thus, the combination of color and luminance differences does not necessarily lead to broader tuning. Adaptation of isoluminant colored gratings (Bradley et al., 1988) indicated tuning that is bandpass but very broad (3.8 octaves) and with a marked falling off at the high-frequency 
end (10 cpd). McCollough-effect tuning appears to fall between that of luminant and isoluminant gratings in that it is of intermediate bandpass and its sensitivity extends at least to $16 \mathrm{cpd}$.

Bradley et al. (1988) examined the proposition that there are two different populations of channels mediating the luminant and isoluminant functions. They tested this hypothesis by cross-adaptation studies; they found very weak cross-adaptation effects confined to high levels of contrast. These results imply that there are two relatively independent groups of channels for nonisoluminant and isoluminant gratings. Bradley et al. (1988) did not accept that conclusion because of their earlier data showing strong cross-masking effects (K. K. De Valois \& Switkes, 1983). Instead, they proposed that there are similar processing mechanisms for color- and luminancecontrast patterns in the early stages of the human visual system.

A learning hypothesis has been put forward to explain the McCollough effect (Murch, 1976). It is argued that the long duration of the McCollough effect (Jones \& Holding, 1975) cannot be accounted for by either an adaptation or a fatigue-like process acting on a neural or sensory mechanism. However, simple adaptation effects can also be quite long lasting, up to $2 \mathrm{~h}$ (Blakemore et al., 1973; Magnussen \& Greenlee, 1985), and these effects are not easily accommodated by a learning model. Murch (1976) developed a classical-conditioning model for the McCollough effect, in which the color of the grating is the unconditioned stimulus (UCS) and the grating is the conditioned stimulus (CS). By continual pairings of these CSs and UCSs, the test gratings somehow obtain the ability to evoke the complementary color of the CAE. The failure to find CAEs with gratings separated by 1 octave is a considerable problem for a learning theory explanation, because all subjects could discriminate between the adapting stimuli on the basis of either color or spatial frequency. That is, why is there no learning to the pairings of clearly discriminable CSs and UCSs?

Dodwell and Humphrey (1990) proposed a functional role for the McCollough effect based on an error-correcting mechanism derived from the adaptation-level (AL) principle of Helson (1964). They argue that there is normally zero correlation between color and orientation, but adaptation with red verticals, for example, shifts the "neutral point" for red toward the end of the red continuum. Thus, the presentation of an achromatic test stimulus is now on the green side of the new AL. While this theory has some general application, the results in our experiments support an explanation based on the adaptation of an orientation detector broadly tuned for spatial-frequency and opponent processes for color (a detailed analysis of this mechanism is set out in our companion paper; (see Webster, Day, Gillies, \& Crassini, 1992). We consider that the McCollough effect gives an insight into the connection between mechanisms relating color to orientation, rather than having an explicit functional role in perception along some color continuum.
Finally, the broad spatial-frequency tuning found for the McCollough effect raises problems for those studies that report spatial-frequency effects using colored checkerboard stimuli to induce a McCollough effect (Green, Corwin, \& Zemon, 1976; May \& Matteson, 1976). In these studies, CAEs were found only at the orientation of the fundamentals of the checkerboards (Kelly, 1976), not at the orientation of edges. It is difficult to understand how such results could occur when tuning of the McCollough effect is 2 octaves and greater. This problem was the starting point for the experiments reported by Webster et al. (1992).

\section{REFERENCES}

Blakemore, C., \& Campbell, F. W. (1969). On the existence of neurones in the human visual system selectively sensitive to the orientation and size of retinal images. Journal of Physiology, 202, 237-260.

Blakemore, C., Muncey, J. P. J., \& Ridley, R. M. (1973). Stimulus specificity in the human visual system. Vision Research, 13, 1915-1931.

Bradley, A., Switkes, E., \& De VAlois, K. K. (1988). Orientation and spatial frequency selectivity of adaptation to color and luminance gratings. Vision Research, 28, 841-856.

Breitmeyer, B. G., COOPER, L. A. (1972). Frequency-specific color adaptation in the human visual system. Perception \& Psychophysics, 11, 95-96.

De Valors, K. K., \&Witkes, E. (1983). Simultaneous masking interactions between chromatic and luminance gratings. Joumal of the Optical Society of America, 73, 11-18.

De Valois, R. L., Albrecht, D. G., \&horell, L. G. (1982). Spatial frequency selectivity of cells in macaque visual cortex. Vision Research, 22, 545-559.

De Valois, R. L., \& De VAlois, K. K. (1980). Spatial vision. Annual Review of Psychology, 31, 309-341.

Dodwell, P. C., \& HuMphrey, G. K. (1990). A functional theory of the McCollough effect. Psychological Review, 97, 78-89.

Green, M., Corwin, T., \& Zemon, V. (1976). A comparison of Fourier analysis and feature analysis in pattern-specific color aftereffects. Science, 192, 147-148.

HeLson, H. (1964). Adaptation level theory: An experimental and systematic approach to behavior. New York: Harper Row.

Henning, G. B., Hertz, B. G., Broadbent, D. F. (1975). Some experiments bearing on the hypothesis that the visual system analyses spatial patterns in independent bands of spatial frequency. $V i$ sion Research, 15, 887-897.

IsHIHARA, S. (1964). Tests for colour-blindness. Tokyo: Kanehara Shuppan.

Jones, P. D., \& Holding, D. H. (1975). Extremely long-term persistence of the McCollough effect. Journal of Experimental Psychology: Human Perception \& Performance, 1, 323-327.

JONES, R. M., \& TulunAY-KeESEY, U. (1975). Local retinal adaptation and spatial frequency channels. Vision Research, 15, 1239-1244.

Kelly, D. H. (1976). Pattern detection and the two-dimensional Fourier transform: Flickering checkerboards and chromatic mechanisms. Vision Research, 16, 277-287.

Kelly, D. H. \& BuRbeCK, C. A. (1985). Critical problems in spatial vision. Critical Reviews in Biomedical Engineering, 10, 125-177.

Lovegrove, W. J., Over, R. (1972). Color adaptation of spatial frequency detectors in the human visual system. Science, 176, 541-543.

Magnussen, S., \& Greenlee, M. W. (1985). Marathon adaptation to spatial contrast: Saturation in sight. Vision Research, 28, 1409-1411. MARR, D. (1982). Vision. San Francisco: W. H. Freeman.

Marr, D., \& Hildreth, E. (1980). Theory of edge detection. Proceedings of the Royal Society of London, Series B, 207, 187-217.

May, J. G., \& Matteson, H. H. (1976). Spatial frequency: Contingent color aftereffects. Science, 192, 145-147.

McCollough, C. (1965). Color adaptation of edge-detectors in the human visual system. Science, 149, 1113-1114. 
MurCh, G. M. (1976). Classical conditioning of the McCollough effect: Temporal parameters. American Joumal of Psychology, 85, 241-247.

Sachs, M. B., Nachmias, J., Robson, J. G. (1971). Spatial-frequency channels in human vision. Journal of the Optical Society of America, 61, 1176-1186

STromeyer, C. F. (1972). Edge-contingent color aftereffects: Spatial frequency specificity. Vision Research, 12, 717-733.

STROMEYER, C. F. (1978). Form-color aftereffects in human vision. In R. Held, H. W. Leibowtiz, \& H. L. Teuber (Eds.), Perception. Handbook of Sensory Physiology (Vol. 8). New York: SpringerVerlag.

Stromeyer, C. F., DAwson, B. M. (1978). Form-color aftereffects: Selectivity to local luminance contrast. Perception, 7, 407-415.

Sullivan, G. D., Georgeson, M. A., \& OAtley, K. (1972). Channels for spatial frequency selection and the detection of single bars by the human visual system. Vision Research, 12, 383-394.

SwiFT, D. J., \& SMITH, R. A. (1982). An action spectrum for spatialfrequency adaptation. Vision Research, 22, 235-246.
Thомаs, J. P. (1970). Model of the function of receptive fields in human vision. Psychological Review, 77, 121-134.

TYLER, C. W. (1975). Spatial frequency filters in cat visual cortex? $V i-$ sion Research, 15, 303-304.

Webster, W. R., Crassini, B., \& Willengerg, K. (1987). Simultaneous color contrast from McCollough effects is spatially contingent. Perception \& Psychophysics, 41, 402-408.

Webster, W. R., Day, R. H., Gillies, O., Crassini, B. (1992). Spatial-frequency-contingent color aftereffects: Adaptation with twodimensional stimulus patterns. Perception \& Psychophysics, 51, 66-78.

Webster, W. R., DAY, R. H., W WLlenberg, K. (1988). Otientationcontingent aftereffects are determined by real color, not induced color. Perception \& Psychophysics, 44, 43-49.

WYATT, H. J. (1974). Singly and doubly contingent aftereffects involving color, orientation and spatial frequency. Vision Research, 14, 1185-1193.

(Manuscript received June 6, 1990; revision accepted for publication August 19, 1991.) 